\title{
GcvA binding site 1 in the gcvTHP promoter of Escherichia coli is required for GcvA-mediated repression but not for GcvA-mediated activation
}

\author{
Laura D. Wonderling, Mark L. Urbanowski and George V. Stauffer
}

Author for correspondence: George V. Stauffer. Tel: +1 319335 7791. Fax: +1 3193359006. e-mail: george-stauffer@uiowa.edu

Department of Microbiology, The University of lowa, lowa City, IA 52242, USA
GcvA binds to three sites in the gcvTHP control region, from base -34 to -69 (site 1), from base -214 to -241 (site 2) and from base -242 to -271 (site 3). Previous results suggested that sites 3 and 2 are required for both GcvAdependent activation and repression of a gcvT:: lacz fusion. However, the results were less clear as to the role of site 1. To determine the role of site 1 in regulation, single and multiple base changes were made in site 1 and tested for their ability to alter GcvA-mediated activation and GcvA/GcvR-mediated repression. Several of the mutants were also tested for effects on GcvA binding to site 1 and the ability of GcvA to bend DNA at site 1. The results are consistent with site 1 playing primarily a role in negative regulation of the gcvTHP operon.

Keywords: gcvTHP, gcvT::lacZ, GcvA, GcvR, repression

\section{INTRODUCTION}

The Escherichia coli glycine cleavage (GCV) enzyme system catalyses the cleavage of glycine to $\mathrm{CO}_{2}, \mathrm{NH}_{3}$, and a one-carbon (C1) methylene unit that is transferred to tetrahydrofolate (Kikuchi, 1973). The resulting 5,10methylenetetrahydrofolate is required as a donor of methyl groups in many biosynthetic reactions and for the methylation of DNA and proteins (Marinus, 1996; Mudd \& Cantoni, 1964). Strains defective in the GCV reaction excrete glycine (Plamann et al., 1983), and strains that overexpress the GCV enzyme system are partial glycine auxotrophs (L. Stauffer, unpublished results), suggesting that the GCV reaction balances the levels of glycine and $\mathrm{C} 1$ units produced according to the needs of the cell. Four enzymes are required for the GCV reaction. Three are encoded by the gcvTHP operon located at $62.5 \mathrm{~min}$ on the E. coli map (Plamann et al., 1983; Stauffer et al., 1994) and the fourth enzyme is encoded by the $l p d$ gene at $2.5 \mathrm{~min}$ (Steiert et al., 1990).

Regulation of the gcvTHP operon is complex, involving both global-acting and $g c v$-specific regulatory proteins (Fig. 1). Lrp, a global regulator involved in regulation of

Abbreviations: CRP, cyclic AMP receptor protein; GCV, glycine cleavage; RNAP, RNA polymerase; WT, wild-type. amino acid metabolic pathways (Calvo \& Matthews, 1994), is required for expression of a $g c v T:: \operatorname{lac} Z$ fusion (Lin et al., 1992; Stauffer \& Stauffer, 1994). Lrp binds to and protects from DNase I digestion a large region of DNA upstream of the $g c v$ promoter from about base -92 to -229 (Stauffer \& Stauffer, 1994). In addition, Lrp binding to this region results in a sharp bend in the gcv DNA (Stauffer \& Stauffer, 1998a). Its primary role may be structural, facilitating formation of stereospecific nucleoprotein complexes required to either activate or repress the $g c v$ operon (Stauffer \& Stauffer, 1999).

PurR, a repressor of several operons involved in nucleotide metabolism (Meng \& Nygaard, 1990; Rolfes \& Zalkin, 1988), represses gcv T : lacZ expression twofold when cells are grown in the presence of purines (Stauffer \& Stauffer, 1994; Wilson et al., 1993a). PurR binds to a site from about base -3 to +17 relative to the transcription initiation site, and possibly interferes with RNA polymerase (RNAP) binding to the $g c v$ promoter (Wilson et al., 1993a).

The cAMP receptor protein (CRP) has recently been shown to play a positive role in controlling $g c v T:: l a c Z$ expression (Wonderling \& Stauffer, 1999). CRP binds to a site from about base -324 to -303 , and although its specific mechanism for regulating $g c v T:: \operatorname{lac} Z$ ex- 


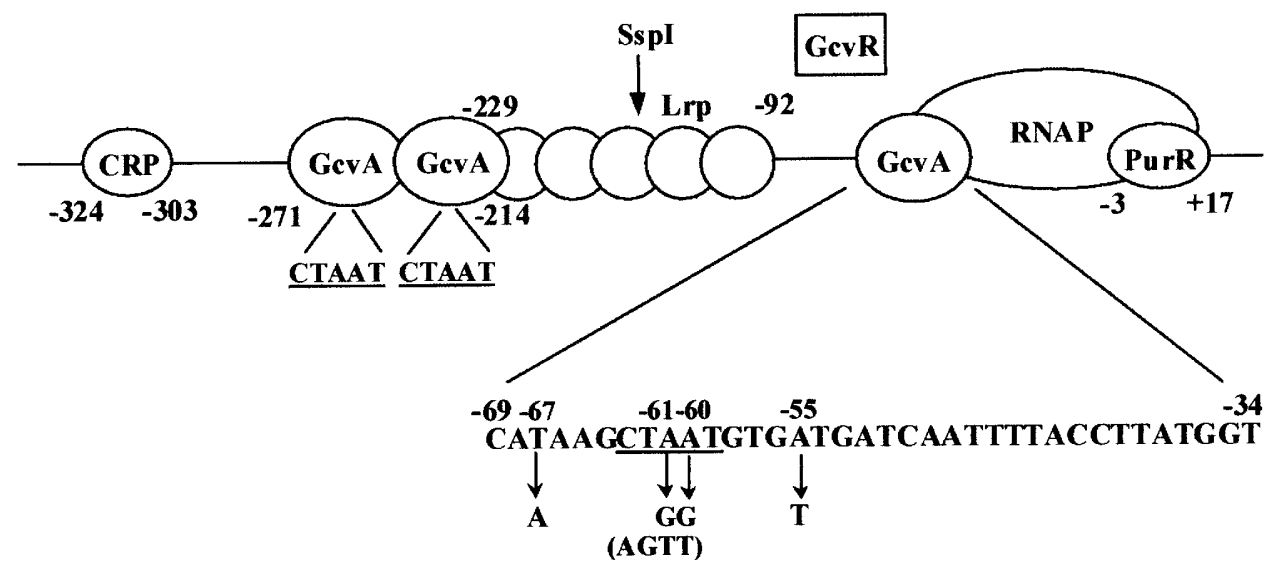

Fig. 1. Proteins known to regulate gcvTHP transcription. The relative binding sites for GcvA (Wilson et al., 1995), Lrp (Stauffer \& Stauffer, 1994 ), CRP (Wonderling \& Stauffer, 1999) and PurR (Wilson et al., 1993a) are shown with respect to the +1 transcription start site. The regulatory molecules are represented as circles or rectangles for simplicity. The exact number of Lrp binding sites is unknown. The underlined nucleotides represent the only sequence present in all three GcvA binding sites. The arrows indicate mutations introduced into site 1. The nucleotides in parentheses (AGTT) depict mutations created in site 1 in a previous study and used in a DNA bending experiment in this study (Fig. 3). The Sspl site used to prepare DNA fragments for gel mobility-shift assays is indicated.

pression has not yet been identified, CRP likely interferes with repression by the GcvA protein.

The GcvA protein is a member of the LysR family of activators that share amino acid homology and domain structure (Schell, 1993). GcvA binds to three sites in the $g c v$ control region, from base -34 to -69 (site 1), from base -214 to -241 (site 2) and from base -242 to -271 (site 3) (Fig. 1) (Wilson et al., 1995). GcvA acts as an activator of $g c v$ expression when glycine is present in the growth medium, causing a six- to sevenfold induction (Wilson et al., 1993b). GcvA also represses the $g c v$ operon over a fivefold range when the purine inosine is added to the growth medium (Wilson et al., 1993a). In the presence of both glycine and purines, the activator function of GevA is dominant (Stauffer \& Stauffer, 1994). Activation and repression by GcvA requires Lrp, presumably to bend the DNA appropriately (Stauffer \& Stauffer, 1998a, b, 1999). Mutations in GcvA have been isolated that affect either the activation or repression function without altering its DNA-binding capabilities (Jourdan \& Stauffer, 1998), suggesting that these two activities lie in separate functional regions.

The GcvR protein is required for repression by GcvA (Ghrist \& Stauffer, 1995). A Tn10 insertion in the gcvR gene causes high constitutive levels of gcvT::lacZ expression due to the loss of GcvA-mediated repression, and overexpression of $g c v R$ leads to superrepression of the fusion that also requires the GcvA protein (Ghrist \& Stauffer, 1995). Preliminary evidence suggests that GcvR interacts directly with GcvA rather than binding $g c v$ DNA (A. Ghrist, unpublished results), but the mechanism by which GcvR causes repression is unknown.

Previous studies suggested that all three GcvA binding sites are required for repression by GcvA, but only sites 2 and 3 are necessary for activation (Wilson et al., 1995).
However, interpretation of the role of site 1 was complicated because the single site 1 allele examined only reduced GcvA binding to site 1 about twofold, and also resulted in a twofold promoter-down phenotype. A prediction based on these early studies is that mutations in site 1 that prevent GcvA binding should affect GcvAmediated repression without interfering with GcvAmediated activation. Such mutations would be useful in understanding the mechanism of the GcvA/GcvRmediated repression system. In this study, mutations were created in GcvA binding site 1 to determine which nucleotides are necessary for GcvA binding and the effects of these mutations on GcvA-mediated repression and activation. The results are consistent with the previous hypothesis that GcvA site 1 is primarily involved in GcvA-mediated repression. The results also suggest that GcvA has different requirements for binding at sites 2 and 3 compared to site 1 .

\section{METHODS}

Strains and plasmids. The E. coli K-12 strains used all carry thi, pheA905, lacU169, araD129 and rpsL150 mutations. In addition, GS852 is purR:: Tn10 (Kilstrup et al., 1989), GS986 is purR::Tn10 and gcvA1 (Wilson et al., 1993a), and GS1053 is $g c v R:: \operatorname{Tn} 10$ (Ghrist \& Stauffer, 1995). Plasmid pMC1403 is a lac fusion vector that has been previously described (Casadaban et al., 1980). Plasmid pGS239 is a derivative of pMC1403 that carries a wild-type (WT) gcvT::lacZ translational fusion (Stauffer et al., 1993). The single-copy plasmid pGS441 carries the gcvAF31L positive control allele and was described previously (Jourdan \& Stauffer, 1998) All other plasmids used are described in the text.

Media. The glucose minimal (GM) medium used was the minimal salts medium of Vogel \& Bonner (1956) supplemented with $0.4 \%$ glucose. Supplements were added at the following concentrations in $\mu \mathrm{g} \mathrm{ml} l^{-1}$ : thiamin, 1 ; phenylalanine, 50; glycine, 300; inosine, 50; ampicillin, 100; tetra- 
cycline, 10. GM medium was always supplemented with phenylalanine and thiamin since all strains used carry the phe A905 and thi mutations.

Enzyme assays. Cells for enzyme assays were harvested from mid-exponential phase cultures $\left(\mathrm{OD}_{600} \sim 0 \cdot 6\right)$. $\beta$-Galactosidase assays were performed as described by Miller (1992), using the chloroform/SDS lysis procedure. All results are the means of two or more assays with each reaction performed in triplicate.

Site-directed mutagenesis and construction of lysogens. Using plasmid pGS239 as template, the PCR 'megaprimer' mutagenesis procedure (Sarkar \& Sommer, 1990) was used to create nucleotide changes in GcvA binding site 1 in the $g c v$ control region. The specific base changes were verified by DNA sequence analysis. The approximately 5400 bp EcoRI$\mathrm{M} f e \mathrm{I}$ fragments carrying mutant $g c v T:$ :lacZ fusions along with the lac Y and lacA genes were isolated from each plasmid and ligated into the EcoRI site of phage $\lambda \mathrm{gt} 2$ (Panasenko et al., 1977). The phages generated were purified as single plaques and designated $\lambda g c v T:: \operatorname{lac} Z(-55 \mathrm{~T}), \operatorname{\lambda gcv} T:: \operatorname{lac} Z(-60 \mathrm{G})$, $\lambda g c v T:: \operatorname{lac} Z(-61 \mathrm{G}), \quad \lambda g c v T:: \operatorname{lac} Z(-67 \mathrm{~A})$ and $\lambda g c v T::$ $\operatorname{lac} Z(-60 \mathrm{G},-61 \mathrm{G},-67 \mathrm{~A})$. The designations in parentheses after the fusions indicate the nucleotide changes and positions relative to the +1 transcription start site. The gcv $T:: \operatorname{lacZ}(1-\mathrm{A})$ and $g c v T:: \operatorname{lac} Z(1-\mathrm{B})$ fusions have four and six base changes, respectively, in site 1 and are described in Fig. 4. Appropriate strains were lysogenized with the above phages and the lysogens verified to carry a single copy of $\lambda$ by infection with phage $\lambda c 190 c 17$ (Shimada et al., 1972).

Gel mobility-shift assay. The purified GcvA protein used in this study was described previously (Jourdan \& Stauffer, 1998). It incorporates six histidine residues at the C-terminal end and shows normal regulation in vivo. The gel mobilityshift assay was carried out essentially as described (Fried \& Crothers, 1981; Garner \& Revzin, 1981). The 464 bp SspIBamHI fragments from plasmid pGS239 and its derivatives were labelled with ${ }^{32} \mathrm{P}$ at the BamHI ends using T4 polynucleotide kinase (Sambrook et al., 1989). The labelled DNA fragments $(<65 \mathrm{pM})$ were included in $20 \mu \mathrm{l}$ reactions containing DNA-binding buffer $(10 \mathrm{mM}$ Tris $/ \mathrm{HCl}, \mathrm{pH} 7 \cdot 0$, $50 \mathrm{mM} \mathrm{KCl}, 0.5 \mathrm{mM}$ EDTA, 5 (v/v) \% glycerol, $1 \mathrm{mM}$ DTT) and $125 \mu \mathrm{g}$ bovine serum albumin $\mathrm{ml}^{-1}$. Reaction mixtures were incubated for $5 \mathrm{~min}$ at $37^{\circ} \mathrm{C}$, and $2 \mu \mathrm{l}$ purified GcvA diluted in DNA binding buffer was added to each mixture as indicated in Figs 2, 3 and 5. Incubation was continued at $37{ }^{\circ} \mathrm{C}$ for $15 \mathrm{~min}, 1 \mu \mathrm{l}$ loading buffer $(0 \cdot 1 \%$ xylene cyanol and $50 \%$ $(\mathrm{v} / \mathrm{v})$ glycerol in $\mathrm{H}_{2} \mathrm{O}$ ) was added, and the samples were loaded onto a $5 \%$ polyacrylamide $/ 3 \%$ glycine gel and electrophoresed at approximately $12 \mathrm{~V} \mathrm{~cm}^{-1}$. The gels were transferred to Whatman $3 \mathrm{MM}$ paper, dried and autoradiographed. The autoradiographs were quantified by densitometry analysis at the University of Iowa Central Research Facility.

DNA bending assay. The DNA bending assay was essentially as described by Kim et al. (1989) using vector pBEND2. The GcvA binding site 1 from either pGS239 or mutant derivatives was amplified by a PCR reaction that added flanking XbaI restriction sites to each end, and the resulting $51 \mathrm{bp} \mathrm{XbaI}$ fragments were cloned into the $X b a I$ site of vector pBEND2. Restriction digests confirmed that all cloned fragments were in the same orientation in pBEND2. For the bending assays, 176 bp MluI, XhoI and BamHI fragments were generated from the WT and mutant plasmids with the GcvA binding site 1 sequence located at specific distances from the centre of the fragment (see Fig. 3). These fragments were labelled with ${ }^{32} \mathrm{P}$ at both ends using T4 polynucleotide kinase (Sambrook et al., 1989). Gel mobility-shift assays were performed as described above and in Fig. 3.

\section{RESULTS}

\section{Point mutations in the conserved 5'-CTAAT-3' sequence of GcvA binding site 1 alter GcvA-mediated repression}

There is little sequence conservation among the GcvA binding sites except for a short 5'-CTAAT-3' sequence (Wilson et al., 1995). In a previous study, four of the five conserved bases in the CTAAT sequence were changed simultaneously in site 1 of the $g c v T:: l a c Z$ control region, causing a partial loss of GcvA-mediated repression, but having no significant effect on GcvAmediated activation (Wilson et al., 1995). However, these changes also caused a GcvA-independent decrease in $g c v T:$ : lacZ expression, complicating interpretation of the results. In the present study, four individual point mutations were created by PCR 'megaprimer' mutagenesis (see Methods) in site 1 at positions $-55,-60$, -61 and -67 relative to the transcription start site $(+1)$ with the expectation that at least one of these mutations would affect GcvA-mediated regulation without affecting promoter strength. Nucleotides -60 and -61 are located in the conserved sequence $5^{\prime}$-CTAAT$3^{\prime}$, and nucleotides -55 and -67 are located at positions that may serve as end points of the $T-N_{11}-A$ motif for binding of GcvA (Fig. 1). The DNA fragments carrying these nucleotide changes were cloned as gcv $T$ : : lac Z fusions into bacteriophage $\lambda \mathrm{gt} 2$ and used to lysogenize strains GS852 and GS986. GS852 is a purR strain, and purine-mediated repression of $\mathrm{gcv}$ in this strain is due solely to the GcvA/GcvR regulatory system (Wilson et al., 1993a). Strain GS986 is a gcvA purR double mutant used as a control since no purine repression of gcv $T:$ : lacZ occurs in GS986, providing a basal level of $\beta$-galactosidase activity for comparison (Wilson et al., 1993a). The lysogens were grown in GM medium supplemented with the purine inosine, and $\beta$ galactosidase levels were measured. In the GS852 WT $\lambda g c v T$ : : lacZ lysogen, growth in GM + inosine resulted in 4.4-fold repression of gcv $T$ : : lacZ expression compared to the GS986 lysogen (Table 1, compare rows 1 and 6). The $\beta$-galactosidase level in the GS852 $\operatorname{lgcvT}:$ : lacZ(-55T) lysogen was slightly higher than the WT lysogen. In the GS852 gcvT::lacZ(-60G), (-61G) and (-67A) lysogens, gcv $T$ : : lacZ expression was twoto threefold higher than in the WT lysogen. In the GS986 gcvT:lacZ(-55T), (-60G), (-61G) and ( $-67 \mathrm{~A})$ lysogens, $\lambda g c v T$ : : lacZ expression was not significantly different from the GS986 WT lysogen. The results suggest that the higher levels of gcvT::lacZ expression of the mutant lysogens in GS852 are primarily due to the loss of GcvA-mediated repression.

Although the above results suggest that the mutations in site 1 interfere with the ability of GcvA to repress $g c v T:: l a c Z$, it is also possible that the mutations created a promoter that allows better GcvA-dependent acti- 
Table 1. Effects of site 1 mutations in GS852 (purR), GS986 (gcvA purR) and GS1053 (gcvR)

GS852 and GS986 lysogens were grown in GM+inosine and GS1053 lysogens were grown in GM, and assayed for $\beta$ galactosidase activity [expressed in Miller units (Miller, 1992)]. All standard deviations were within $13 \%$ of the mean.

\begin{tabular}{|lc|}
\hline Strain & $\begin{array}{c}\boldsymbol{\beta} \text {-Galactosidase } \\
\text { activity }\end{array}$ \\
\hline GS852 $\lambda g c v T:: \operatorname{lacZ}$ & 24 \\
GS852 $\lambda g c v T:: \operatorname{lacZ}(-55 \mathrm{~T})$ & 34 \\
GS852 $\lambda g c v T:: \operatorname{lacZ}(-60 \mathrm{G})$ & 66 \\
GS852 $\lambda g c v T:: \operatorname{lacZ}(-61 \mathrm{G})$ & 82 \\
GS852 $\lambda g c v T:: \operatorname{lacZ}(-67 \mathrm{~A})$ & 51 \\
GS986 $\lambda g c v T:: \operatorname{lacZ}$ & 107 \\
GS986 $\lambda g c v T:: \operatorname{lacZ}(-55 \mathrm{~T})$ & 104 \\
GS986 $\lambda g c v T:: \operatorname{lacZ}(-60 \mathrm{G})$ & 107 \\
GS986 $\lambda g c v T:: \operatorname{lacZ}(-61 \mathrm{G})$ & 133 \\
GS986 $\lambda g c v T:: \operatorname{lacZ}(-67 \mathrm{~A})$ & 107 \\
GS1053 $\lambda g c v T:: \operatorname{lacZ}$ & 1681 \\
GS1053 $\lambda g c v T:: \operatorname{lacZ}(-55 \mathrm{~T})$ & 1773 \\
GS1053 $\lambda g c v T:: \operatorname{lacZ}(-60 \mathrm{G})$ & 2130 \\
GS1053 $\lambda g c v T:: \operatorname{lacZ}(-61 \mathrm{G})$ & 1630 \\
GS1053 $\lambda g c v T:: \operatorname{lacZ}(-67 \mathrm{~A})$ & 1886 \\
\hline
\end{tabular}

Table 2. Effects of a triple base change in site 1 in GS852 (purR) and GS986 (gcvA purR)

$\beta$-Galactosidase activity is expressed in Miller units (Miller, 1992). All standard deviations were within $11 \%$ of the mean.

\begin{tabular}{|c|c|c|c|}
\hline \multirow[t]{2}{*}{ Strain } & \multicolumn{3}{|c|}{$\begin{array}{l}\beta \text {-Galactosidase activity of cells } \\
\text { grown in GM with }\end{array}$} \\
\hline & No addition & Glycine & Inosine \\
\hline GS852 $\lambda g c v T:: l a c Z$ & 133 & 879 & 27 \\
\hline $\begin{array}{l}\text { GS852 } \lambda g c v T:: l a c Z \\
(-60 \mathrm{G},-61 \mathrm{G},-67 \mathrm{~A})\end{array}$ & 320 & 861 & 78 \\
\hline GS986 igcvT::lacZ & 84 & 90 & 79 \\
\hline $\begin{array}{l}\text { GS986 } \lambda g c v T:: l a c Z \\
(-60 \mathrm{G},-61 \mathrm{G},-67 \mathrm{~A})\end{array}$ & 121 & 126 & 113 \\
\hline
\end{tabular}

vation. Therefore, the WT and the mutant igcv $T: \operatorname{lac} Z(-55 \mathrm{~T}),(-60 \mathrm{G}),(-61 \mathrm{G})$ and $(-67 \mathrm{~A})$ phage were used to lysogenize the $g c v R$ strain GS1053. In this strain, GcvA can no longer repress gcv $T:$ : lacZ expression (Ghrist \& Stauffer, 1995). If the mutations in site 1 allow GcvA to activate better, they might be expected to cause a two- to threefold increase in expression in GS1053 above the levels observed in the WT $\operatorname{gcv} T:$ :lacZ lysogen. However, if the site 1 mutations interfere with the ability of GcvA to repress, then the nucleotide changes should have no effect in GS1053 since GcvA fails to repress in this strain. The GS1053 lysogens carrying the WT and mutant phages were grown in GM medium and $\beta$-galactosidase levels were measured (Table 1). The $-55 \mathrm{~T},-61 \mathrm{G}$ and $-67 \mathrm{~A}$ mutations at site 1 did not cause a significant increase in gcv T: : lacZ expression in GS1053 compared to the WT fusion (Table 1). Although $-60 \mathrm{G}$ caused a slight 1·3fold increase in $\beta$-galactosidase activity compared to the WT fusion, the increase was small compared to the $2 \cdot 7$ fold increase seen in strain GS852. These results suggest that the base changes at positions $-60,-61$ and -67 relative to the transcription initiation site interfere with GcvA-mediated repression rather than activation. However, it is possible that a $g c v R$ strain expresses $g c v T:$ : lacZ at a maximal level, and higher levels may be difficult to achieve.

\section{A three-base mutation in the conserved 5'-CTAAT-3' sequence of site 1 causes a phenotype similar to the single-base changes}

Despite the loss of GcvA-mediated repression caused by the point mutations when the GS852 lysogens were grown in GM+inosine, the levels of gcvT::lacZ expression are still significantly lower than the levels seen in the $g c v R$ strain GS1053 (Table 1). If binding site 1 is required for repression by GcvA and GcvR, why are the levels of expression lower in the site 1 mutants compared to a $g c v R$ mutant? One possibility is the inability of single-base changes to eliminate binding of GcvA to site 1. To test this possibility, a triple mutant was constructed, combining the changes at bases $-60,-61$ and -67 in a single gcvT: : lacZ fusion. This fusion was cloned into the $\lambda \mathrm{gt} 2$ vector and the resulting phage, designated $\lambda g c v T:$ : $\operatorname{lac} Z(-60 \mathrm{G},-61 \mathrm{G},-67 \mathrm{~A})$ was used to lysogenize strains GS852 and GS986. Cells were grown in GM, GM+glycine or GM+inosine and $\beta$ galactosidase levels were measured (Table 2). Expression of the gcv $T:$ : lacZ ( - 60G, -61G, - 67A) fusion was two- to threefold higher in GS852 compared to the WT gcvT: : lacZ fusion when cells were grown in either GM or GM + inosine. The mutations in site 1 had no significant effect on $\beta$-galactosidase expression when the lysogen was grown in GM+glycine. In the gcv A purR strain GS986, the site 1 triple mutant no longer caused a two- to threefold increase in $g c v T:$ : lacZ expression compared to WT, although there was a slight but reproducible increase $(\sim 1 \cdot 4$-fold). Since GS986 is a gcvA strain, these results indicate that the site 1 mutations cause a small GcvA-independent effect, probably an increase in promoter strength. Despite this small GcvA-independent effect, the majority of the increase seen in GS852 can be attributed to a loss of GcvAmediated repression. However, the site 1 triple mutant did not completely relieve GcvA-mediated repression of the $g c v T:$ :lacZ fusion as seen in a $g c v R$ strain, but exhibited a phenotype similar to the single-base mutants.

\section{Gel mobility-shift assay}

Gel mobility-shift assays were performed to determine whether GcvA binds to the triple mutant site 1 with a reduced affinity compared to the WT site 1 . The WT 


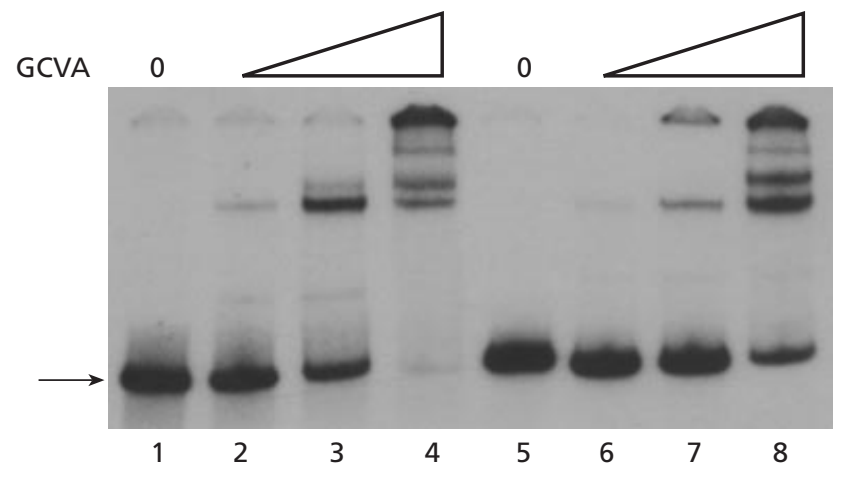

Fig. 2. Mobility-shift assay for binding of GcvA to WT gcvT::lacZ and the triple site 1 mutant gcvT::lacZ $(-60 \mathrm{G},-61 \mathrm{G},-67 \mathrm{~A})$. The WT Sspl-BamHI fragment was used for lanes 1-4, and the mutant site 1 Sspl-BamHI fragment was used for lanes 5-8. The concentration of GcvA dimer added to each reaction is as follows: lanes 1 and 5, $0 \mathrm{nM}$; lanes 2 and $6,21.5 \mathrm{nM}$; lanes 3 and 7, $43 \mathrm{nM}$; lanes 4 and 8 , $86 \mathrm{nM}$. The arrow indicates the position of the unbound fragment.

template was a $464 \mathrm{bp}$ SspI-BamHI fragment from pGS239 and the mutant template was an identical $464 \mathrm{bp}$ SspI-BamHI fragment except that it carries the $-60 \mathrm{G}$, $-61 \mathrm{G}$ and $-67 \mathrm{~A}$ mutations. These two gcv fragments lack the upstream GcvA binding sites 2 and 3 (see Fig. 1) and allow assessment of the binding of GcvA to site 1 in the absence of the higher affinity sites 2 and 3 , eliminating the additional complexes formed when all three binding sites are present (Wilson et al., 1995). At $21.5 \mathrm{nM}$ GcvA dimer, a small amount of the WT fragment was bound by GcvA and shifted to two bands of slower mobility (Fig. 2). At $43 \mathrm{nM} \mathrm{GcvA}$ dimer, more of the WT fragment was bound, most of which was shifted to one band. In addition, a third band of slower mobility was seen at this concentration of GcvA. Nearly all of the WT fragment was bound at $86 \mathrm{nM}$ dimer and several bands of slower mobility appeared (Fig. 2, lane 4). The mutant site 1 DNA fragment showed a mobility shift pattern essentially identical to that seen with the WT fragment, except that the binding affinity of GcvA for the mutant site 1 fragment is about twofold lower than its affinity for the WT fragment (Fig. 2, lanes 5-8).

\section{The mutations in the conserved sequence of site 1 are involved in DNA conformation}

Recent experiments demonstrated that GcvA bends DNA when it binds to any of the three binding sites in the gcv control region (G. Stauffer, unpublished results). Since the mutations in the conserved $5^{\prime}$-CTAAT-3' sequence of site 1 alter repression to varying degrees but reduce GcvA's binding affinity less than twofold, it is possible that some other function, such as DNA bending, is affected by mutations in the region that leads to altered regulation. To test this possibility, the triple site 1 mutant $-60 \mathrm{G},-61 \mathrm{G},-67 \mathrm{~A}$, the four-base mutant

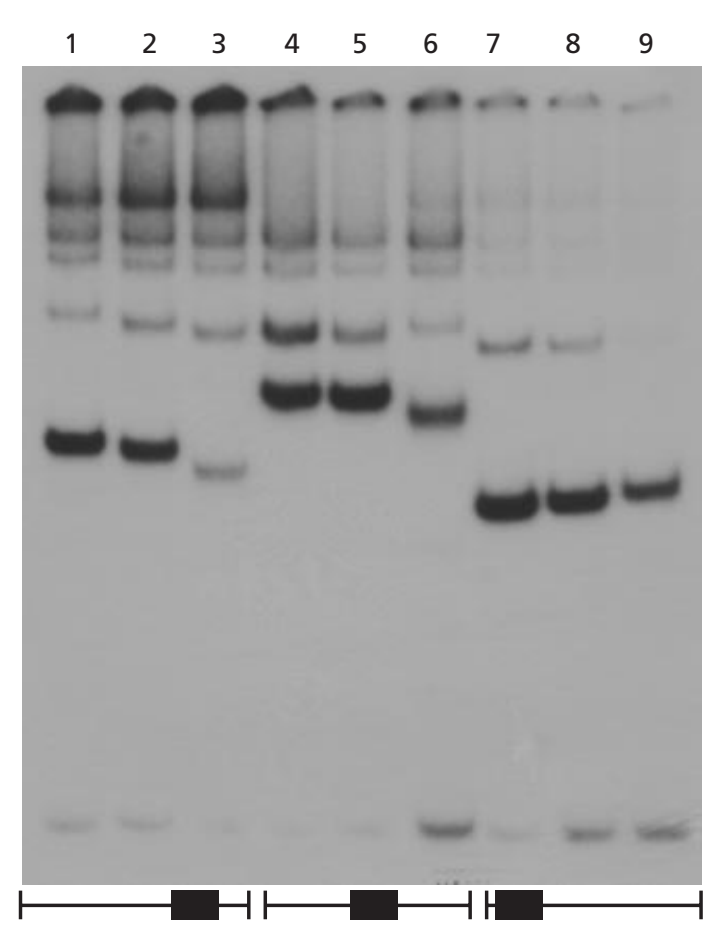

Fig. 3. DNA bending assays using WT site 1 (lanes 1, 4, and 7), the triple site 1 mutant (lanes 2, 5, and 8), and a previously described four-base site 1 mutant fragment (see Fig. 1) (lanes 3,6 , and 9). GcvA was added to all reactions at a final concentration of $52 \mathrm{nM}$ dimer. The relative locations of the GcvA binding site with respect to the ends of each fragment are shown at the bottom of the figure. The binding site, shown as a black box, is located near the right end of the fragments in lanes 1-3, near the centre of the fragments in lanes 4-6, and near the left end of the fragments in lanes 7-9.

site 1 described previously (Wilson et al., 1995) (Fig. 1) and the WT site 1 were cloned into the vector pBEND2. The plasmids were digested with restriction enzymes to generate equal length DNA fragments with site 1 located at varying distances from the centre of the fragments (Fig. 3). These fragments were then used as templates in gel mobility-shift assays. Since protein-induced DNA bending alters the end-to-end distance of a fragment, a determinant of mobility through a polyacrylamide gel, DNA bending was detected by a change in mobility when site 1 was located at different distances from the centre of the fragment. As expected, when the WT site 1 was located at the right end or the left end of the fragment, it showed a faster mobility than when site 1 was located in the centre of the fragment (Fig. 3, lanes 1, 4 and 7). The mobilities of the two mutant fragments were faster than the WT fragment when the target site was located at the right end of the fragment (Fig. 3, lanes $1-3)$, but slower when the target sites were located at the left end of the fragment (lanes 7-9). When the target sites were located near the middle of the DNA fragment, the three-base mutant fragment exhibited a similar mobility to the WT and the four-base mutant fragment exhibited a faster mobility than the WT fragment (lanes 


\title{
WT sequence -69-CATAAG CTAATGTGATGATCAATTTTACCTTATGGT--34 \\ Site 1-A oligo \\ Site 1-B oligo

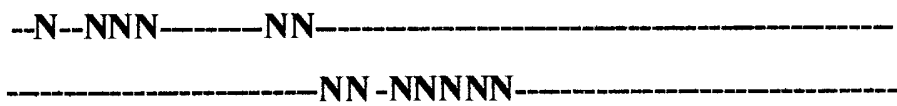

Mutant (1-A)

CATATTCTAAGCTGATGATCAATTTTACCTTATGGT $-65-64 \quad-59-58$

Mutant (1-B)

\author{
CATAAGCTAATG GCATCCTGAATTTTACCTTATGGT \\ $-57-56 \quad-53-52-50$
}

Fig. 4. The oligonucleotides site 1-A and site 1-B were used in PCR 'megapriming' reactions to incorporate random nucleotides at the positions indicated by ' $N$ '. The dashed lines indicate that WT sequence is incorporated at these positions. The boxed nucleotides represent those conserved among WT GcvA binding sites 1, 2 and 3. The underlined nucleotides are the specific mutations present in the site 1 sequences of the 1-A and 1-B mutants.

4-6). The results of the DNA bending assays show that the mutations cause some disruption of the bending at site 1.

\section{Isolation of a GcvA site 1 that relieves repression}

Since previous mutations (Wilson et al., 1995) and the mutations described above did not completely relieve GcvA/GcvR-mediated repression of $g c v T:: l a c Z$, the role of site 1 in regulation is difficult to ascertain. To further characterize site 1 , a partially random mutagenesis was performed that could potentially alter site 1 at six different nucleotides in the region surrounding the conserved 5'-CTAAT- $3^{\prime}$ sequence, or at seven positions promoter proximal to the conserved sequence (Fig. 4). This was accomplished by the use of two oligonucleotides, referred to as oligo site 1-A and oligo site 1$\mathrm{B}$, that have any of the four nucleotides incorporated at bases $-58,-59,-64,-65,-66,-68$ or at bases $-50,-51,-52,-53,-54,-56$ and -57 relative to the transcription initiation site at +1 . Using a second WT primer, a pool of PCR products was generated, the fragments were cloned into pMC1403 to reconstruct gcv T: : lacZ fusions and used to transform GS852. One transformant from each selection was sequenced to determine the nucleotide changes. The transformants isolated using oligo 1-A and oligo 1-B had four and five base changes, respectively (Fig. 4).

To test for their abilities to relieve repression by GcvA, the 1-A and 1-B fragments were cloned as $\lambda g c v T:: \operatorname{lac} Z$ fusions and used to lysogenize strains GS852 and GS986. The resulting lysogens were grown in GM, GM + glycine and $\mathrm{GM}+$ inosine, and assayed for $\beta$-galactosidase activity. The 1-A mutations had little effect when compared to the WT gcvT: lacZ fusion in purR strain GS852 (Table 3, compare rows 1 and 2). However, in the gcvA purR strain GS986, the 1-A mutations caused a two- to threefold decrease in expression compared to the WT fusion, indicating that one or more of the altered
Table 3. The effects of the 1-A and 1-B mutations on gcvT::lacZ expression in GS852 (purR) and GS986 ( $g c v A$ purR)

$\beta$-Galactosidase activity is expressed in Miller units (Miller, 1992). All standard deviations were within $19 \%$ of the mean.

\begin{tabular}{|lccc|}
\hline Strain & \multicolumn{3}{c|}{$\begin{array}{c}\boldsymbol{\beta} \text {-Galactosidase activity of cells } \\
\text { grown in GM with }\end{array}$} \\
\cline { 2 - 4 } & No addition & Glycine & Inosine \\
\hline GS852 $\lambda g c v T:: \operatorname{lac} Z$ & 158 & 914 & 20 \\
GS852 $\lambda g c v T:: \operatorname{lac} Z(1-\mathrm{A})$ & 212 & 760 & 27 \\
GS852 $\lambda g c v T:: \operatorname{lacZ}(1-\mathrm{B})$ & 293 & 863 & 130 \\
GS986 $\lambda g c v T:: \operatorname{lac} Z$ & 122 & 102 & 84 \\
GS986 $\lambda g c v T:: \operatorname{lac} Z(1-\mathrm{A})$ & 38 & 42 & 32 \\
GS986 $\lambda g c v T:: \operatorname{lacZ}(1-\mathrm{B})$ & 41 & 48 & 36 \\
\hline
\end{tabular}

nucleotides in the 1-A mutant are important for general promoter strength (compare rows 4 and 5). In addition, these nucleotides might be important for normal gcvT:: lacZ expression since any effect on GcvAmediated regulation may be masked by the promoterdown effect. The 1-B fusion had about twofold higher levels of $g c v T:$ :lacZ expression than the WT fusion when cells were grown in GM and over a sixfold increase in GM + inosine. There was no significant effect of the mutations in GM+glycine. These results suggest that the mutations present in the 1-B fusion interfere with negative regulation, especially in the presence of inosine (Table 3, compare rows 1 and 3). It should be noted that the 1-B mutations also caused a two- to threefold decrease in $g c v T:$ :lacZ expression when compared to the WT gcvT::lacZ fusion in GS986, indicating that in the absence of GcvA these mutations decrease the basal levels of expression (compare rows 4 and 6). This GcvA-independent decrease may indicate 
Table 4. GcvA is unable to repress $g c v T::$ lac $Z$ when the $1-B$ mutations are present in site 1

Cells were grown in GM + inosine and assayed for $\beta$-galactosidase activity (expressed in Miller units) (Miller, 1992). All standard deviations were within $7 \%$ of the mean, except where indicated.

\begin{tabular}{|llc|}
\hline Strain & Relevant genotype & $\begin{array}{c}\boldsymbol{\beta} \text {-Galactosidase } \\
\text { activity }\end{array}$ \\
\hline GS986 $\lambda g c v T:: l a c Z$ & $g c v A$ purR & 100 \\
GS986 $\lambda g c v T:: \operatorname{lacZ}+$ pGS441 & $g c v A$ purR $+g c v A F 31 L$ & $10^{*}$ \\
GS986 $\lambda g c v T:: \operatorname{lacZ}(1-\mathrm{B})$ & $g c v A$ purR & 40 \\
GS986 $\lambda g c v T:: \operatorname{lacZ}(1-\mathrm{B})+\mathrm{pGS441}$ & $g c v A$ purR $+g c v A F 31 L$ & 30 \\
\hline
\end{tabular}

*Value is the average of 9 and 11 units.

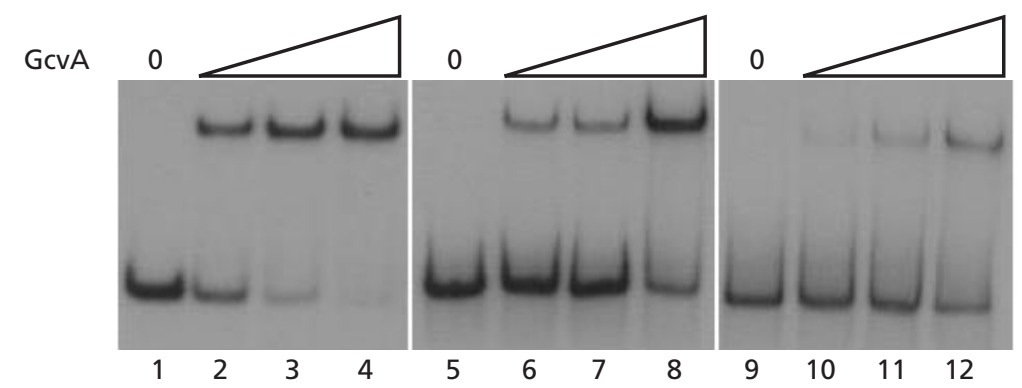

Fig. 5. Mobility-shift assay for binding of GcvA to the 464 bp BamHI-Sspl WT, 1-A and 1-B fragments. GcvA was added at the following dimer concentrations: lanes 1, 5 and 9, $0 \mathrm{nM}$; lanes 2,6 and 10, $16 \mathrm{nM}$; lanes 3,7 and $11,32 \mathrm{nM}$; lanes 4, 8 and 12, $64 \mathrm{nM}$.

that the 1-B mutations cause an even larger relief of repression than reflected by the $\beta$-galactosidase levels in Table 4, that is concealed by the GcvA-independent promoter decrease. However, it is possible that the promoter-down effect exhibited by the 1-B mutations only occurs in the absence of GcvA, perhaps by interfering with RNAP binding to the promoter. This possibility is difficult to test since attempts at DNase I protection assays with RNAP and $g c v$ DNA have proven unsuccessful.

An alternative interpretation of the results is that the 1$B$ changes have enhanced the ability of GcvA to activate rather than interfere with its ability to repress. We tested this hypothesis using the gcvAF31L allele. The gcvAF31L mutation is a positive control mutation (Jourdan \& Stauffer, 1998). The altered amino acid at position 31 of GcvA presumably prevents an interaction with RNAP that is required for activation of $g c v T::$ lacZ; however, GcvA-F31L is still able to repress gcv T:: lacZ normally. We tested the effects of the gcvAF31L allele on the expression of $g c v T:: \operatorname{lacZ}(1-\mathrm{B})$. GS986 $\lambda g c v T:: \operatorname{lac} Z$ and GS986 $\lambda g c v T:: \operatorname{lac} Z(1-B)$ were transformed with the single-copy plasmid carrying the gcvAF31L allele and $\beta$-galactosidase levels were measured (Table 4). Expression of the WT fusion is repressed 10-fold in the presence of the gcvAF31L allele. As noted above, in the absence of $g c v A$ the 1-B fusion shows decreased expression when compared to the WT fusion due to the promoter-down effect (Table 4, compare rows 1 and 3). In the presence of GcvA-F31L, the $g c v T:: \operatorname{lacZ}(1-\mathrm{B})$ fusion is repressed only $1 \cdot 3$-fold (compare rows 3 and 4 ).

\section{Binding of GcvA to the site 1-A and site 1-B mutants}

The 464 bp BamHI-SspI fragments, identical to those described in the mobility-shift assays above except for the base changes, were isolated from the 1-A and 1-B mutants, ${ }^{32} \mathrm{P}$-labelled at the BamHI ends and used in gel mobility-shift assays. The 1-A mutant site 1 fragment was able to bind GcvA with approximately a twofold decreased affinity compared to WT site 1 , suggesting that the nucleotides at positions $-57,-58,-64$ and -65 have some role in GcvA binding (Fig. 5, compare lanes 1-4 with 5-8). The 1-B mutant fragment showed at least a fourfold decrease in affinity for GcvA, indicating that one or more of the nucleotides altered are important for binding of GcvA (Fig. 5, compare lanes 1-4 with 9-12). Although the 1-B mutations did not completely abolish binding by GcvA, the 1-B mutant is the first site 1 mutant to exhibit more than a twofold decrease in binding affinity for GcvA, suggesting that the central region of site 1 is important for GcvA binding, rather than the conserved 5'-CTAAT-3' sequence important for recognition and binding of GcvA at sites 2 and 3 (Wilson et al., 1995).

To separate the GcvA-dependent and -independent effects of the 1-B mutations, site-directed mutagenesis was used to create single-base changes at positions -50 , $-52,-53,-56$ and -57 . We expected that one or more of these nucleotides would be required for GcvA binding and repression, and for general promoter strength independent of GcvA. The fragments containing the single-base changes were verified by DNA sequencing and tested for their effects on these various 
Table 5. Effects on expression of WT and $g c v T:: / a c Z(1-B)$ fusions when $g c v R$ is overexpressed in GS852 (purR) and GS986 (gcvA purR)

Cells were grown in GM medium and assayed for $\beta$-galactosidase activity (expressed in Miller units) (Miller, 1992). All standard deviations were within $8 \%$ of the mean.

\begin{tabular}{|c|c|c|}
\hline Strain & Relevant genotype & $\begin{array}{c}\beta \text {-Galactosidase } \\
\text { activity }\end{array}$ \\
\hline GS852 $\lambda g c v T:: \operatorname{lacZ}$ & purR & 162 \\
\hline GS852 igcvT::lacZ + pGS334 & $\operatorname{purR}\left(g c v R^{+++}\right)$ & 58 \\
\hline GS852 $\lambda g c v T:: \operatorname{lac} Z(1-\mathrm{B})$ & purR & 225 \\
\hline GS852 $\lambda g c v T:: \operatorname{lac} Z(1-B)+$ pGS334 & $\operatorname{purR}\left(g c v R^{+++}\right)$ & 142 \\
\hline GS986 $\lambda g c v T:: \operatorname{lac} Z$ & gcvA purR & 88 \\
\hline GS986 igcvT::lacZ + pGS334 & $g c v A p u r R\left(g c v R^{+++}\right)$ & 89 \\
\hline GS986 igcvT::lacZ(1-B) & gcvA purR & 36 \\
\hline GS986 $\lambda g c v T:: \operatorname{lac} Z(1-\mathrm{B})+\mathrm{pGS} 334$ & $g c v A p u r R\left(g c v R^{+++}\right)$ & 31 \\
\hline
\end{tabular}

functions. Surprisingly, none of the single-base changes had any significant effect on regulation by GcvA, general promoter strength or the ability to bind GcvA (data not shown).

\section{Superrepression by multi-copy $g c v R$ in the presence of the 1-B mutations in site 1}

Our current model proposes that GcvA binding to site 1 is required for GcvA-mediated repression in the presence of inosine. However, we do not know if the repression mediated by the GcvR protein requires the GcvA/site 1 interaction or if GcvR represses through a separate mechanism. To determine if repression by GcvR is affected by the mutations at site 1, the multi-copy plasmid pGS334 that overexpresses $g c v R$ and causes superrepression of a WT fusion was transformed into GS852 $\lambda g c v T:: l a c Z$, GS852 $\lambda g c v T:: \operatorname{lacZ}(1-\mathrm{B})$, GS986 $\lambda g c v T:: \operatorname{lac} Z$ and GS986 $\lambda g c v T:: \operatorname{lac} Z(1-B)$. If the 1-B mutations at site 1 interfere with repression by GcvR, then superrepression should be antagonized by the nucleotide changes. However, if site 1 is involved in a secondary mechanism of repression that does not involve GcvR, then superrepression should occur in the presence of multi-copy $g c v R$. The transformants were grown in GM medium and assayed for $\beta$-galactosidase activity. Plasmid pGS334 repressed the WT gcvT: :lacZ fusion nearly $2 \cdot 8$-fold (Table 5 , rows 1 and 2 ) and the gcvT: : lacZ (1-B) fusion about 1.6-fold (rows 3 and 4). GS986 served as the control strain to demonstrate that multi-copy $g c v R$ cannot cause repression in the absence of GcvA. Thus, the 1-B mutations eliminate most of the GcvR-mediated superrepression, suggesting that repression by GcvR requires an intact site 1 .

\section{DISCUSSION}

GcvA-mediated repression of the $g c v$ operon has been suggested to require GcvA binding to site 1 (Wilson et al., 1995). This study demonstrates that the sequence requirement at site 1 is complex. Single and multiple base changes throughout the potential $\mathrm{T}-\mathrm{N}_{11}-\mathrm{A}$ sequence, which includes the conserved 5'-CTAAT-3' sequence of site 1 (Fig. 1), relieve repression by GcvA about two- to threefold in the purR strain GS852 (Table 1 ), but show less than a twofold decrease in GcvA binding affinity compared to WT site 1 (Fig. 2). It is likely that the loss of repression is due in part to a loss of GcvA binding to site 1 . In addition, the three and four base site 1 mutations examined show altered GcvAinduced DNA bending at site 1 (Fig. 3). Thus, an altered bend in the DNA may in some way also be responsible for part of the loss of repression. DNA bending by the phage $\phi 29 \mathrm{p} 4$ protein has been shown to be important in its ability to act as a repressor to hinder RNAP binding (Rojo \& Salas, 1991). Of interest is that the three-base mutant isolated in this study runs more slowly than the four-base mutant isolated previously (Wilson et al., 1995) when site 1 is at the right end of the DNA fragment, but faster when located at the left end of the fragment (Fig. 3). One interpretation to explain the gelshift pattern is that the base changes alter the centre of the bend angle as well as or instead of the degree of bending.

The most severe allele examined that alters both GcvA binding and repression is the 1-B mutant, with five base changes (Fig. 4). These changes result in about a fourfold decrease in GcvA binding affinity in vitro when compared to WT site 1 (Fig. 5), and in vivo caused a sixfold loss of GcvA-mediated repression when cells were grown in GM+inosine (Table 3). The mutations also caused a two- to threefold decrease in promoter strength (Table 3). However, when the five bases were changed individually, none of the changes had a significant effect on $g c v T::$ lacZ expression or on the binding affinity of GcvA, again suggesting that several bases must be altered to interfere with binding and repression by GcrA.

The 1-A and 1-B mutants isolated in this study, as well as the four-base mutant isolated previously (Wilson et al., 1995), showed GcvA-independent promoter-down 
effects (Table 3). It should be noted that the promoterdown effects are only observed in the absence of GcvA. Thus, it is possible that RNAP interacts with this region of $g c v$ in the absence of GcvA to maintain basal promoter activity, and the mutations interfere with this interaction. It is known that the C-terminal domain of the $\alpha$ subunit of RNAP interacts with up-elements located upstream of the promoter -35 sequence element (Estrem et al., 1999). Although a sequence is present in $g c v$ from base -44 to -54 that shows a 7 out of 11 base match with the proposed up-element consensus distal subsite (Fig. 1), this degree of homology is considered insignificant as a subsite (Estrem et al., 1999). Furthermore, the 1-A mutant and the four-base mutant (Wilson et al., 1995) lie upstream of this sequence. We have been unsuccessful at footprinting RNAP at the $g c v$ promoter to verify an RNAP-DNA interaction in this region.

Previous studies showed that overexpressing $g c v R$ favours repression of a $g c v T$ : : lacZ fusion even under activating conditions (glycine supplementation), and overexpressing gcvA favours activation even under repressing conditions (purine supplementation) (Ghrist \& Stauffer, 1998). Since GcvA activates a gcv T::lacZ fusion in the absence of GcvR, and GcvR-mediated repression is dependent on a functional GcvA protein, a model was proposed that GcvA homo-oligomers activate the $g c v$ operon, whereas GcvA/GcvR heterooligomers repress the $g c v$ operon (Ghrist \& Stauffer, 1995). The small co-regulators glycine and purines possibly determine whether the activator or the repressor form of GcvA is favoured in the cell. We believe our results are consistent with this model. Since GcvA/GcvR, in the presence of inosine, represses a $g c v T$ : : lac Z fusion four- to fivefold in a site 1-dependent manner, GcvA binding at site 1 in the presence of GcvR appears to be necessary to repress transcription. Furthermore, as none of the changes have a significant effect on activation, site 1 appears necessary only for repression. However, the role(s) of GcvR in the repression mechanism is still unclear. Also unclear are the different sequence requirements for site 1 compared to sites 2 and 3. It is possible that the difference is related to the mechanism GcvA employs to switch between activation and repression.

\section{ACKNOWLEDGEMENTS}

This investigation was supported by Public Health Service grant GM26878 from the National Institute of General Medical Science.

\section{REFERENCES}

Calvo, J. M. \& Matthews, R. G. (1994). The leucine-responsive regulatory protein, a global regulator of metabolism in Escherichia coli. Microbiol Rev 58, 466-490.

Casadaban, M. J., Chou, J. \& Cohen, S. N. (1980). In vitro gene fusions that join an enzymatically active $\beta$-galactosidase segment to amino-terminal fragments of exogenous proteins: Escherichia coli plasmid vectors for the detection and cloning of translational initiation signals. J Bacteriol 143, 971-980.
Estrem, S. T., Ross, W., Gaal, T., Chen, Z. W. S., Niu, W., Ebright, R. H. \& Gourse, R. L. (1999). Bacterial promoter architecture: subsite structure of UP elements and interactions with the carboxy-terminal domain of the RNA polymerase $\alpha$ subunit. Genes Dev 13, 2134-2147.

Fried, M. \& Crothers, D. M. (1981). Equilibria and kinetics of $l a c$ repressor-operator interactions by polyacrylamide gel electrophoresis. Nucleic Acids Res 9, 6505-6525.

Garner, M. M. \& Revzin, A. (1981). A gel electrophoresis method for quantifying the binding of proteins to specific DNA regions: application to components of the Escherichia coli lactose operon regulatory system. Nucleic Acids Res 9, 3047-3060.

Ghrist, A. C. \& Stauffer, G. V. (1995). Characterization of the Escherichia coli $g c v R$ gene encoding a negative regulator of $g c v$ expression. J Bacteriol 177, 4980-4984.

Ghrist, A. C. \& Stauffer, G. V. (1998). Promoter characterization and constitutive expression of the Escherichia coli gcvR gene. J Bacteriol 180, 1803-1807.

Jourdan, A. D. \& Stauffer, G. V. (1998). Mutational analysis of the transcriptional regulator GcvA: amino acids important for activation, repression, and DNA binding. J Bacteriol 180, $4865-4871$.

Kikuchi, G. (1973). The glycine cleavage system: composition, reaction mechanism, and physiological significance. Mol Cell Biochem 1, 169-187.

Kilstrup, M., Meng, L. M., Neuhard, J. \& Nygaard, P. (1989). Genetic evidence for a repressor of synthesis of cytosine deaminase and purine biosynthesis enzymes in Escherichia coli. J Bacteriol 171, 2124-2127.

Kim, J., Zwieb, C., Wu, C. \& Adhya, S. (1989). Bending of DNA by gene-regulatory proteins : construction and use of a DNA bending vector. Gene 85, 15-23.

Lin, R., D'Ari, R. \& Newman, E. B. (1992). placMu insertions in genes of the leucine regulon: extension of the regulon to genes not regulated by leucine. J Bacteriol 174, 1948-1955.

Marinus, M. G. (1996). Methylation of DNA. In Escherichia coli and Salmonella: Cellular and Molecular Biology, pp. 782-791. Edited by F. C. Neidhardt \& others. Washington, DC: American Society for Microbiology.

Meng, L. M. \& Nygaard, P. (1990). Identification of hypoxanthine and guanine as the co-repressors for the purine regulon genes of Escherichia coli. Mol Microbiol 4, 2187-2192.

Miller, J. H. (1992). A Short Course in Bacterial Genetics. Cold Spring Harbor, NY: Cold Spring Harbor Laboratory.

Mudd, S. H. \& Cantoni, G. L. (1964). Biological transmethylation, methyl-group neogenesis and other 'one-carbon' metabolic reactions dependent upon tetrahydrofolic acid. In Comprehensive Biochemistry, pp. 1-47. Edited by M. Florkin \& E. H. Stotz. Amsterdam: Elsevier.

Panasenko, S. M., Cameron, J. R., Davis, R. W. \& Lehman, I. R. (1977). Five-hundredfold overproduction of DNA ligase after induction of a hybrid lambda lysogen constructed in vitro. Science 196, 188-189.

Plamann, M. D., Rapp, W. D. \& Stauffer, G. V. (1983). Escherichia coli K12 mutants defective in the glycine cleavage enzyme system. Mol Gen Genet 192, 15-20.

Rojo, R. \& Salas, M. (1991). A DNA curvature can substitute phage $\phi 29$ regulatory protein $\mathrm{p} 4$ when acting as a transcriptional repressor. EMBO J 10, 3429-3438.

Rolfes, R. J. \& Zalkin, H. (1988). Escherichia coli gene purR encoding a repressor protein for purine nucleotide synthesis. $J$ Biol Chem 263, 19653-19661. 
Sambrook, J., Fritsch, E. F. \& Maniatis, T. (1989). Molecular Cloning: a Laboratory Manual, 2nd edn. Cold Spring Harbor, NY: Cold Spring Harbor Laboratory.

Sarkar, G. \& Sommer, S. S. (1990). The 'megaprimer' method of site-directed mutagenesis. BioTechniques 8, 404-407.

Schell, M. A. (1993). Molecular biology of the LysR family of transcriptional regulators. Annu Rev Microbiol 47, 597-626.

Shimada, K., Weisberg, R. A. \& Gottesman, M. E. (1972). Prophage lambda at unusual chromosomal locations. I. Location of the secondary attachment sites and the properties of the lysogens. $J$ Mol Biol 63, 483-503.

Stauffer, L. T. \& Stauffer, G. V. (1994). Characterization of the $g c v$ control region from Escherichia coli. J Bacteriol 176, 6159-6164.

Stauffer, L. T. \& Stauffer, G. V. (1998a). Roles for GcvA-binding sites 3 and 2 and the Lrp-binding region in $g c v T:$ : lacZ expression in Escherichia coli. Microbiology 144, 2865-2872.

Stauffer, L. T. \& Stauffer, G. V. (1998b). Spacing and orientation requirements of GcvA-binding sites 3 and 2 and the Lrp-binding region for gcvT::lacZ expression in Escherichia coli. Microbiology 144, 1417-1422.

Stauffer, L. T. \& Stauffer, G. V. (1999). Role for the leucineresponsive regulatory protein (Lrp) as a structural protein in regulating the Escherichia coli gcvTHP operon. Microbiology 145, 569-576.

Stauffer, L. T., Ghrist, A. \& Stauffer, G. V. (1993). The Escherichia coli $g c v T$ gene encoding the T-protein of the glycine cleavage enzyme system. DNA Seq-J DNA Seq Mapping 3, 339-346.

Stauffer, L. T., Fogarty, S. J. \& Stauffer, G. V. (1994). Characterization of the Escherichia coli gcv operon. Gene 142, 17-22.

Steiert, P. S., Stauffer, L. T. \& Stauffer, G. V. (1990). The $l p d$ gene product functions as the L protein in the Escherichia coli glycine cleavage enzyme system. J Bacteriol 172, 6142-6144.

Vogel, H. J. \& Bonner, D. M. (1956). Acetylornithinase of Escherichia coli: partial purification and some properties. J Biol Chem 218, 97-106.

Wilson, R. L., Stauffer, L. T. \& Stauffer, G. V. (1993a). Roles of the GcvA and PurR proteins in negative regulation of the Escherichia coli glycine cleavage enzyme system. J Bacteriol 175, 5129-5134.

Wilson, R. L., Steiert, P. S. \& Stauffer, G. V. (1993b). Positive regulation of the Escherichia coli glycine cleavage enzyme system. J Bacteriol 175, 902-904.

Wilson, R. L., Urbanowski, M. L. \& Stauffer, G. V. (1995). DNA binding sites of the LysR-type regulator GcvA in the $g c v$ and $g c v A$ control regions of Escherichia coli. J Bacteriol 177, 4940-4946.

Wonderling, L. D. \& Stauffer, G. V. (1999). The cyclic AMP receptor protein is dependent on GcvA for regulation of the $\mathrm{gcv}$ operon. J Bacteriol 181, 1912-1919.

Received 27 July 2000; accepted 1 August 2000. 